\title{
A Definite Case of L-carbocisteine-induced Pneumonia with CATCH22 Syndrome
}

\author{
Kenichiro Kudo ${ }^{1}$, Eiki Ichihara ${ }^{1}$, Akiko Hisamoto ${ }^{1}$, Katsuyuki Hotta ${ }^{1}$, Nobuaki Miyahara ${ }^{1}$, \\ Yasushi Tanimoto ${ }^{1}$, Sadaharu Akagi ${ }^{3}$, Katsuya Kato ${ }^{4}$, \\ Mitsune Tanimoto $^{2}$ and Katsuyuki Kiura ${ }^{1}$
}

\begin{abstract}
A 32-year-old male with CATCH22 syndrome presented with a high fever and productive cough after taking drugs for acute bronchitis, including L-carbocisteine. Chest radiography revealed ground-glass opacities in the bilateral lung fields. He had a history of similar pneumonia. Under the assumption of drug-induced pneumonia, or bacterial or viral pneumonia, all drugs including L-carbocisteine were discontinued, and antibiotics were started. A drug-induced lymphocyte stimulation test was positive only for L-carbocisteine. The only drug in common between this and the previous episode of pneumonia was L-carbocisteine. We thus concluded that this was a definite case of L-carbocisteine-induced pneumonia in a patient with CATCH22 syndrome.
\end{abstract}

Key words: drug-induced pneumonia, L-carbocisteine, CATCH22 syndrome, drug-induced lymphocyte stimulation test

(Intern Med 52: 97-100, 2013)

(DOI: 10.2169/internalmedicine.52.7882)

\section{Introduction}

Owing to its ability to break disulfide bonds in glycoproteins, L-carbocisteine has been used as a mucolytic agent for adjunctive therapy in respiratory tract disorders characterized by excessive viscous mucus, including chronic obstructive airway disease. Serious toxicities are rare, although StevensJohnson syndrome, hepatic dysfunction, and anaphylactic shock have been reported. Only one case of possible Lcarbocisteine-induced pneumonia has been reported to date (1).

CATCH22 syndrome, which is associated with a chromosome $22 \mathrm{q} 11.2$ deletion, is relatively common, with an incidence of $1 / 3,000$, and presents with micrognathia, congenital heart disease, hypocalcemia, seizures, and cellular immunodeficiency (2).

We herein report a case of L-carbocisteine-induced pneu- monia in a patient with CATCH22 syndrome.

\section{Case Report}

A 32-year-old male presenting with a productive cough was treated with L-carbocisteine, ambroxol, levofloxacin, and 1,3-dimethylxanthine under a diagnosis of acute bronchitis at a local clinic. His symptoms progressed rapidly, with a high fever and productive cough. Chest radiography revealed ill-defined opacities, and he was transferred to our hospital. The patient had a history of $\mathrm{CATCH} 22$ syndrome with tetralogy of Fallot and a similar episode of pneumonia treated in an intensive care unit 3 years earlier. His father died of interstitial lung disease at 50 years of age.

On admission, there was no anemia, jaundice, or clubbed fingers. Coarse crackles and wheezing were heard in both lungs. His initial vital signs were as follows: blood pressure, 123/92 $\mathrm{mmHg}$; heart rate, 107 beats/min; respiration rate,

\footnotetext{
${ }^{1}$ Department of Allergy and Respiratory Medicine, Okayama University Hospital, Japan, ${ }^{2}$ Department of Hematology, Oncology and Respiratory Medicine, Okayama University Graduate School of Medicine, Dentistry and Pharmaceutical Sciences, Japan, ${ }^{3}$ Department of Cardiovascular Surgery, Okayama University Hospital, Japan and ${ }^{4}$ Department of Radiology, Okayama University Hospital, Japan

Received for publication April 3, 2012; Accepted for publication May 29, 2012

Correspondence to Dr. Kenichiro Kudo, kudoken19800411@yahoo.co.jp
} 


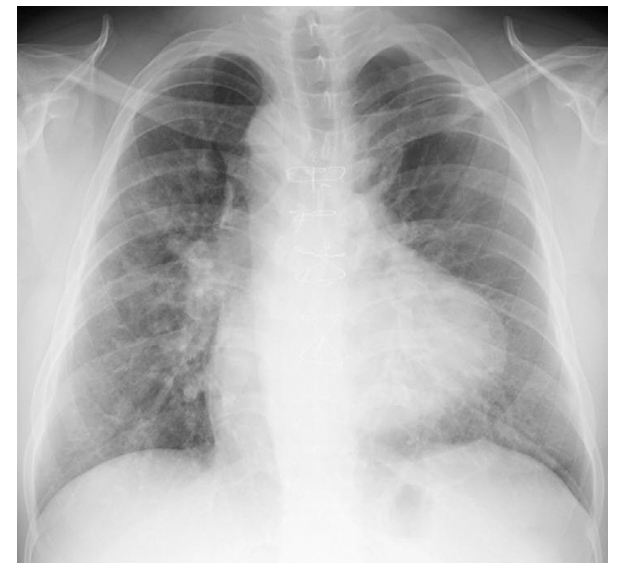

Figure 1. Chest radiographs on admission showed groundglass opacities in bilateral lung fields and a typical bootshaped heart due to tetralogy of Fallot.

$17 / \mathrm{min}$; body temperature, $37.4^{\circ} \mathrm{C}$; and percutaneous arterial oxygen saturation, $91 \%$ in room air. The laboratory findings were as follows: white blood cell count, $15,730 / \mathrm{mm}^{3}$ [neutrophils, 77\% (segmented cells, 67\%; stab cells, 10\%); lymphocytes, 14\%; eosinophils, 0\%]; C-reactive protein, 7.8 $\mathrm{mg} / \mathrm{dL}$; procalcitonin, $0.031 \mathrm{ng} / \mathrm{mL}$; lactase dehydrogenase, $328 \mathrm{IU} / \mathrm{L}$; sialylated carbohydrate antigen KL-6, $343 \mathrm{U} / \mathrm{mL}$; serum surfactant protein $\mathrm{D}, 25 \mathrm{pg} / \mathrm{mL} ; \beta-\mathrm{D}-$ Glucan, $<6.0 \mathrm{pg} /$ $\mathrm{mL}$ : immunoglobulin $\mathrm{G}, 1,469.6 \mathrm{mg} / \mathrm{dL}$, brain natriuretic peptide, $25 \mathrm{pg} / \mathrm{mL}$; and CD4-positive $\mathrm{T}$ lymphocyte count, $538 / \mathrm{mm}^{3}$.

A sputum smear and culture did not reveal any bacteria, fungi, mycobacteria, or Pneumocystis jirovecii. Chest radiography showed ground-glass opacities in bilateral lung fields and a boot-shaped heart typical of tetralogy of Fallot (Fig. 1). Computed tomography (CT) scans of the lungs demonstrated patchy ground-glass opacities with interlobular septal lines (Fig. 2A).

We suspected that the patient had either drug-induced pneumonia or community-acquired pneumonia. Therefore, all drugs, including L-carbocisteine were discontinued, and he was treated with doripenem. The clinical symptoms and laboratory findings improved gradually, and a clear improvement was radiologically evident on a CT scan performed on day 8 after beginning this treatment (Fig. 2B). The stimulatory indices determined by drug-induced lymphocyte stimulation tests (DLSTs) for L-carbocisteine, ambroxol, levofloxacin, and 1,3-dimethylxanthine were $221 \%, 151 \%, 121 \%$, and $108 \%$, respectively, where $>180 \%$ is considered to be a positive result.

The patient had a history of similar pneumonia after taking L-carbocisteine three years earlier. On the previous admission, all drugs (including L-carbocisteine) were discontinued because the patient was intubated due to severe hypoxia. A CT scan performed on the previous admission showed patchy bilateral ground-glass opacities, similar to the CT findings of the present admission (Fig. 2C). At the previous admission, bronchoalveolar lavage (BAL) showed
$50 \%$ recovery of $100 \mathrm{~mL}$ of normal saline, including $35 \times 10^{6}$ cells $/ \mathrm{mL}$ consisting of $60 \%$ alveolar macrophages, $8.7 \%$ eosinophils, $25 \%$ neutrophils, and $5.9 \%$ lymphocytes. Eosinophils were increased in the BAL fluid, which had a CD4/8 ratio of 0.19 . The stimulatory indices determined by DLSTs for cefditoren pivoxil, ceftriaxone sodium, and meropenem trihydrate were $103 \%, 99 \%$, and $103 \%$, respectively; the index for L-carbocisteine was not checked. His clinical symptoms and chest shadows improved after these drugs were stopped and new antibiotics were administered, before steroid treatment. At that time, a diagnosis of cryptogenic organizing pneumonia (COP) was made, although the actual cause of the COP was unknown. L-carbocisteine was the only drug in common between the present admission and the previous event.

\section{Discussion}

To our knowledge, this is the first report of a definite case of drug-induced pneumonia due to L-carbocisteine in a patient with CATCH22 syndrome, although one case of probable L-carbocisteine-induced pneumonia has been reported previously (1). Despite a diagnosis of COP during the previous admission, we did not suspect that L-carbocisteine had produced the severe toxicity, because this agent has been widely and safely used in patients with mucus sputum due to acute bronchitis or chronic obstructive lung disease in Japan since 1981. As this patient had CATCH22 syndrome, we assumed that, during his first admission, he had common community-acquired pneumonia caused by an unidentified bacterial or viral infection and an undiscovered immunodeficiency, rather than drug-induced pneumonia. However, after the second episode, we made a diagnosis of L-carbocisteineinduced pneumonia.

In Japan, there are five criteria for diagnosing druginduced pneumonia. (1) Pneumonitis appears within 1 to 6 weeks after the administration of the drug. (2) The first symptoms of pneumonitis are fever, cough, dyspnea, and rash; cases with two or more of these symptoms are considered positive. (3) There is eosinophilia or leukocytosis in the peripheral blood. (4) The DLST and/or patch test results are positive. (5) Pneumonitis reappears after the chance readministration of the drug (3). Ground-glass opacities on the chest radiograph were detected 13 days after administering L-carbocisteine in the present case, although the symptoms occurred after the development of acute bronchitis. As this case fulfilled at least four criteria (i.e., criteria 1,3, 4, and $5)$, it was considered to be a definite case of drug-induced pneumonia due to L-carbocisteine. A previous case of Lcarbocisteine-induced pneumonia fulfilled only one criterion (positive DLST result). Therefore, this is the first definitive case of L-carbocisteine-induced pneumonia reported to date.

The CT findings in drug-induced pneumonia are categorized as diffuse or multifocal ground-glass opacities with intralobular interstitial thickening; patchy ground-glass opacities with centrilobular opacities and interlobular septal lines; 


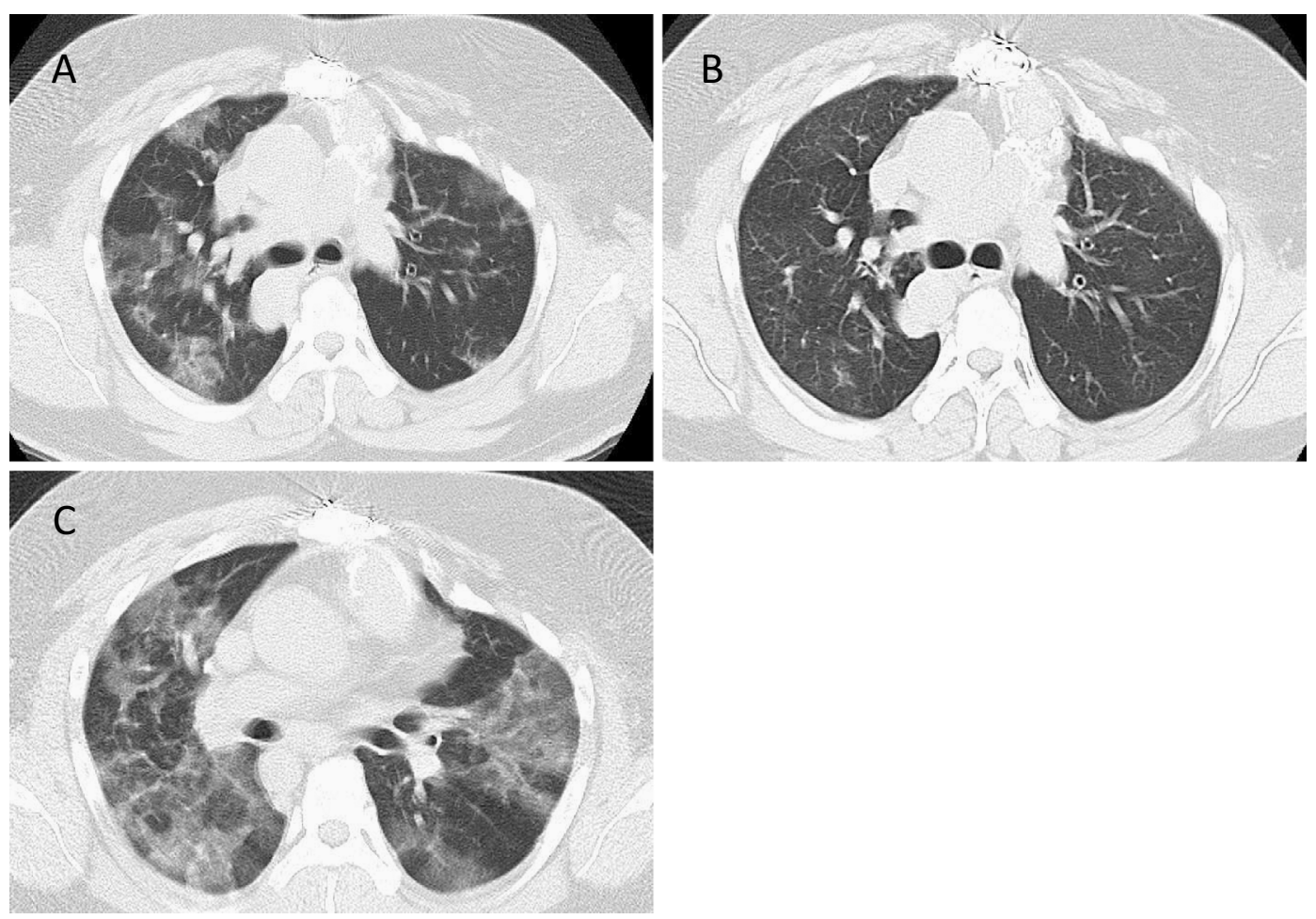

Figure 2. A: A chest CT scan on admission showed patchy ground-glass opacities with interlobular septal lines in both lungs and severe calcification of the aorta. B: The ground-glass opacities on the CT scan were markedly improved 8 days after discontinuing L-carbocisteine. C: A chest CT scan performed during the previous admission showed patchy bilateral ground-glass opacities similar to the CT findings on this admission.

and diffuse ground-glass opacities with patchy consolidation (4). The patchy ground-glass opacities with interlobular septal lines seen on $\mathrm{CT}$ in this case were consistent with drug-induced pneumonia.

The BAL fluid in drug-induced pneumonia shows increased numbers of neutrophils, eosinophils, and/or lymphocytes $(5,6)$. In rats with allergic lung inflammation, neutrophils in the parenchyma and BAL fluid peaked at 24 hours and then declined rapidly, whereas the eosinophil accumulation in BAL fluid peaked at 72 hours (7). The increases in both the eosinophil and neutrophil numbers in the BAL fluid observed in the present case were also compatible with drug-induced pneumonia.

CATCH22 syndrome is associated with cellular immunodeficiency because of anomalies in the thymus, parathyroid, and great vessels (8). The immunological phenotype varies widely among patients. Although severe T lymphocyte immunodeficiency is rare, combined partial immunodeficiency is more common and leads to recurrent sinopulmonary infections (9). In this case, the level of serum immunoglobulin and the number of cells related to cellular immunity were normal. Nevertheless, we cannot exclude that there might have been a recurrent bacterial infection due to an unidentified immunodeficiency in $\mathrm{CATCH} 22$ syndrome in this patient. The relationship between $\mathrm{CATCH} 22$ syndrome and L-carbocisteine-induced pneumonia is unknown.

In conclusion, we herein reported the first definite case of
L-carbocisteine-induced pneumonia in a patient with CATCH22 syndrome. As L-carbocisteine is a widely used mucolytic agent, pneumonia induced by this agent may occur in other cases. Therefore, it is important to take a careful history and perform a DLST in order to make an accurate diagnosis of drug-induced pneumonia.

The authors state that they have no Conflict of Interest (COI).

\section{References}

1. Koreeda Y, Tanoue A, Kumamoto T, et al. A possible case of drug-induced pneumonia due to L-carbocisteine. Nihon Kokyuki Gakkai Zasshi 45: 609-614, 2007 (in Japanese, Abstract in English).

2. Lee Y, Han Y. Aspiration pneumonia in the child with DiGeorge syndrome. Korean J Anesthesiol 60: 449-452, 2011.

3. Tamura M. Drug-induced pneumonitis. Intern Med 22: 262-270, 1983.

4. Akira M, Ishikawa H, Yamamoto S. Drug-induced pneumonitis: thin-section CT findings in 60 patients. Radiology 224: 852-860, 2002.

5. Danel C, Israel-Biet D, Costabal U, et al. Clinical guidelines and indications for bronchoalveolar lavage (BAL): drug induced pneumonitis. Eur Respir J 3: 952-953, 1990.

6. Akoun GM, Cadrenal JL, Milleron BJ, et al. Bronchoalveolar lavage cell data in 19 patients with drug-associated pneumonitis (except amiodarone). Chest 99: 98-104, 1991.

7. Schneider T, Valzen DV, Moqbel BJ, et al. Kinetics and quantita- 
Intern Med 52: 97-100, 2013 DOI: 10.2169/internalmedicine.52.7882

tion of eosinophil and neutrophil recruitment to allergic lung inflammation in a brown Norway rat model. Am J Respir Cell Mol Biol 17: 702-712, 1997.

8. Markert ML, Hummell DS, Rosenblatt HM, et al. Complete Di-
George syndrome: persistence of profound immunodeficiency. J Pediatr 132: 15-21, 1998.

9. Gennery AR. Immunological aspects of 22q11.2 deletion syndrome. Cell Mol Life Sci 69: 17-27, 2012.

(C) 2013 The Japanese Society of Internal Medicine http://www.naika.or.jp/imonline/index.html 